\title{
BRANCH GROUPS, ORBIT GROWTH, AND SUBGROUP GROWTH TYPES FOR PRO- $p$ GROUPS
}

\author{
YIFTACH BARNEA $^{1}$ and JAN-CHRISTOPH SCHLAGE-PUCHTA ${ }^{2}$ \\ ${ }^{1}$ Department of Mathematics, Royal Holloway, University of London, Egham, \\ Surrey TW20 0EX, UK; \\ email: y.barnea@rhul.ac.uk \\ ${ }^{2}$ Institut für Mathematik, Ulmenstr. 69, 18051 Rostock, Germany; \\ email: jan-christoph.schlage-puchta@uni-rostock.de
}

Received 26 February 2019; accepted 9 April 2020

\begin{abstract}
In their book Subgroup Growth, Lubotzky and Segal asked: What are the possible types of subgroup growth of the pro- $p$ group? In this paper, we construct certain extensions of the Grigorchuk group and the Gupta-Sidki groups, which have all possible types of subgroup growth between $n^{(\log n)^{2}}$ and $e^{n}$. Thus, we give an almost complete answer to Lubotzky and Segal's question. In addition, we show that a class of pro- $p$ branch groups, including the Grigorchuk group and the Gupta-Sidki groups, all have subgroup growth type $n^{\log n}$.
\end{abstract}

2010 Mathematics Subject Classification: 20E07 (primary); 20E08, $20 \mathrm{E} 18$ (secondary)

\section{Introduction and results}

For the rest of this paper, $p$ is a fixed prime and $\log n=\log _{p} n$. For a group $G$, we denote by $s_{n}(G)$ the number of subgroups of $G$ of index at most $n$, where if $G$ is a topological group, we mean closed subgroups. The subgroup growth of $G$ is the asymptotic behaviour of $s_{n}(G)$. See Lubotzky and Segal [8] for the history and background of the study of subgroup growth.

Given a function $f: \mathbb{N} \rightarrow \mathbb{R}$, we say that $G$ has subgroup growth of type $f(n)$ if there exist $a, b>0$ such that $s_{n}(G) \leqslant f(n)^{a}$ for all $n$ and $f(n)^{b} \leqslant s_{n}(G)$ for infinitely many $n$. If in addition, $f(n)^{b} \leqslant s_{n}(G)$ for all large $n$, then we say that $G$ has strict subgroup growth of type $f(n)$. If $G$ is a pro- $p$ group, we restrict $n$ to be a power of $p$ in the last condition. Segal in [11] and Pyber in [10] showed that there are finitely generated profinite groups of any 'reasonable' subgroup growth

(C) The Author(s) 2020. This is an Open Access article, distributed under the terms of the Creative Commons Attribution licence (http://creativecommons.org/licenses/by/4.0/), which permits unrestricted re-use, distribution, and reproduction in any medium, provided the original work is properly cited. 
type. On the other hand, Shalev showed in [14] that for a pro- $p$ group $G$ if there exists a constant $c<\frac{1}{8}$ such that $s_{n}(G) \leqslant n^{c \log n}$ for all $n$, then $G$ has polynomial subgroup growth, that is, its subgroup growth is of type $n$. Therefore, for pro- $p$ groups, there is a gap in the subgroup growth between type $n$ and type $n^{\log n}$.

Pro- $p$ groups of subgroup types $n, n^{\log n}$ and $2^{n}$ are found in many natural examples. Segal and Shalev [12] constructed metabelian pro- $p$ groups with types $2^{n^{1 / d}}$ for any $d \in \mathbb{N}$ and Klopsch in [7] constructed metabelian pro- $p$ groups of types $2^{n^{(d-1) / d}}$ for any $d \in \mathbb{N}$; see [8, Ch. 9]. No other types were discovered. Hence, Lubotzky and Segal posed in [8, Open Problems] the following problems:

\section{PROBLEM 1.}

(a) Are there any other gaps in the subgroup growth types of pro-p groups?

(b) What other subgroup growth types occur for pro-p groups?

(c) Is there an uncountable number of subgroup growth types (up to the necessary equivalence) for pro-p groups?

In this paper, we show that all functions in the range $n^{(\log n)^{2}}$ to $e^{n}$ occur as the subgroup growth type of a pro- $p$ group. We give two different constructions of groups. Although the second construction gives the full range, it is not effective and the growth type is not strict. On the other hand, the first construction gives only a countable sequence of types, but it is effective and the subgroup growth type is strict.

THEOREM 1. For every integer $k \geqslant 1$ and prime number $p$, there exists a residually finite p-group $H$ such that $s_{n}(H)$ is of strict type $n^{(\log n)^{k}}$. By taking the pro-p completion of $H$, we obtain a pro-p group with strict subgroup growth type $n^{(\log n)^{k}}$.

The second construction is less effective, and gives inferior bounds, but applies to a larger range of functions.

THEOREM 2. Let $f: \mathbb{N} \rightarrow \mathbb{N}$ be a function such that $f(n) \geqslant n^{3}$ and $\frac{f(n)}{p^{n}} \rightarrow 0$. Then there exists a residually finite p-group $H$ such that $s_{n}(H)$ is of type $e^{f(\log n)}$. By taking the pro- $p$ completion of $H$, we obtain a pro- $p$ group with subgroup growth type $e^{f(\log n)}$.

Note that this means that we can obtain any subgroup growth type between $n^{(\log n)^{2}}=e^{(\log n)^{3}}$ and $e^{n}=e^{p^{\log n}}$ excluding $e^{n}$. However, we of course obtain 
subgroup growth type $e^{n}$ by considering a non-Abelian free pro- $p$ group. Hence, the only questions left considering the type are the following.

PROBLEM 2. What types of subgroup growth of pro- $p$ groups exist between $n^{\log n}$ and $n^{(\log n)^{2}}$ ? In particular, is there a gap in the growth types?

We comment that one way of attacking this problem is to better understand the canonical permutation module of certain branch groups; see Proposition 5 and the remarks thereafter.

Comparing Theorem 1 with Theorem 2, we pose the following problem.

PROBLEM 3. Let $f$ be a function satisfying the assumptions of Theorem 2. Give an analytic condition which implies that there exists a pro-p group $G$ of strict growth type $f$. For example, is it true that every log-concave function, that is, a function $f$ satisfying $(\log f)^{\prime \prime}<0$, is the strict growth type of a pro- $p$ group? Is it at least true for all functions $f(n)=e^{n^{\alpha} \log ^{\beta} n}$, where $0 \leqslant \alpha<1$ ?

It is not hard to see from Proposition 3(3) that the subgroup growth of a pro- $p$ group cannot vary too wildly. We are not sure whether the answer to the second part is positive, that is, we will not be surprised if there exists a log-concave function $f$ such that there are no pro- $p$ groups of strict growth type $f$. However, we believe that the answer to the third part is positive, that is, for any $0 \leqslant \alpha<1$ and $\beta$, there is a pro- $p$ group of strict growth type $e^{n^{\alpha} \log ^{\beta} n}$.

For a group $G$, we denote by $d(G)$ the minimal number of generators of $G$, where if $G$ is a topological group, we mean topological generators. We write $\Phi_{p}(G)=[G, G] G^{p}$, where $G^{p}$ is the (closed) subgroup generated by the $p$ powers in $G$. We denote $d_{p}(G)=d\left(G / \Phi_{p}(G)\right)=\operatorname{dim}_{\mathbb{F}_{p}}\left(G / \Phi_{p}(G)\right)$. Note that if $G$ is a pro- $p$ group, then $\Phi_{p}(G)$ is its Frattini subgroup and $d(G)=d_{p}(G)$. Note also that if $\widehat{G}$ is the pro- $p$ completion of $G$, then $d(G) \geqslant d(\widehat{G})=d_{p}(\widehat{G})=$ $d_{p}(G)$. We denote by $d_{G}(m)$ the maximum of $d(U)$, as $U$ ranges over all subgroups of index $p^{m}$, where if $G$ is a topological group, we mean closed subgroups. Similarly, for a group $G$, we denote by $d_{p, G}(m)$ the maximum of $d_{p}(U)$, as $U$ ranges over all (closed) subgroups of index $p^{m}$. In the case where it is clear from the context to which $p$ and to which $G$ we refer, we will omit the subscripts $p$ and $G$.

For a pro- $p$ group $G$, the asymptotic behaviour of $s_{p^{m}}(G)$ is intimately linked to the asymptotic behaviour of $d_{G}(m)$. We say that $G$ has logarithmic p-rank gradient, if $\alpha(G)=\lim _{m \rightarrow \infty} \frac{d_{p}(m)}{m}$ exists, is finite and positive. Combining [8, Proposition 1.6.2] and [8, Lemma 4.2.1] yields the following. 
PROPOSITION 3. Let $G$ be a p-group or a pro-p group.

(1) If $G$ has logarithmic p-rank gradient $\alpha$, then

$$
p^{\frac{\alpha^{2}}{4(\alpha+1)} m^{2}+o\left(m^{2}\right)} \leqslant s_{p^{m}}(G) \leqslant p^{\frac{\alpha}{2} m^{2}+o\left(m^{2}\right)} .
$$

(2) If $H$ is a p-group or a pro-p group, respectively, commensurable with $G$, and $G$ has logarithmic rank gradient $\alpha$, then $H$ also has logarithmic p-rank gradient $\alpha$.

(3) If $\mu \leqslant d_{p}(m-\mu)$, then

$$
p^{\mu\left(d_{p}(m-\mu)-\mu\right)} \leqslant s_{p^{m}}(G) \leqslant p^{\sum_{v=1}^{m-1} d(v)} .
$$

Our constructions of residually finite $p$-groups with new types are extensions of the well-known Grigorchuk group and the Gupta-Sidki groups. These groups have served as examples displaying quite unexpected behaviour for various properties; most famously, the Grigorchuk group has intermediate (word) growth. They are infinite residually finite $p$-groups and belong to the class of branch groups. They and their pro- $p$ completions are important examples of just infinite groups, that is, their only nontrivial quotients are finite. Although they have been extensively studied, the subgroup growth of these groups has not been determined up until now. However, it was a common belief that they will provide a new type of subgroup growth for pro- $p$ groups. Nevertheless, in Theorem 9 , we will prove that a more general class of groups has subgroup growth of type $n^{\log n}$. In particular, we will obtain the following corollary.

COROLlARY 4. If $G$ is the Grigorchuk group, then

$$
2^{\frac{9}{40} m^{2}+o\left(m^{2}\right)} \leqslant s_{2^{m}}(G) \leqslant 2^{6 m^{2}+o\left(m^{2}\right)} .
$$

If $G$ is the Gupta-Sidki p-group with $p \geqslant 3$, then

$$
p^{\frac{1}{8} m^{2}+o\left(m^{2}\right)} \leqslant s_{p^{m}}(G) \leqslant p^{\frac{3 p^{2}-4 p+1}{2} m^{2}+o\left(m^{2}\right)} .
$$

The apparent discrepancy between the lower and upper bounds for the subgroup growth mostly stems from our poor knowledge about the size of $\alpha(G)$. In theory, arbitrarily good approximations to $\alpha(G)$ are computable. However, the naïve approach fails due to a huge amount of memory needed. We therefore ask the following.

PRoblem 4. Give a numerical approximation to $\alpha(G)$, where $G$ is the Grigorchuk or a Gupta-Sidki group. 
We comment that previously known examples of pro- $p$ groups with subgroup growth type $n^{\log n}$ are of different nature, that is, either linear and analytic groups or the Nottingham group and its index subgroups. Furthermore, many of these examples are just infinite. Ershov and Jaikin-Zapirain in [2] constructed new hereditarily just infinite pro- $p$ groups. Moreover, these groups have subgroup growth type at least $n^{(\log n)^{2-\epsilon}}$, where $\epsilon$ is any positive number (private communication). However, no additional information is known about the subgroup growth type of these examples. We therefore suggest the following problem.

PROBLEM 5. What are the possible subgroup growth types of just infinite prop groups? In particular, compute the subgroup growth types of the examples constructed by Ershov and Jaikin-Zapirain. Furthermore, can a just infinite prop group have exponential subgroup growth type?

Our constructions depend on the fact that the Grigorchuk group and the GuptaSidki groups act on each element in the boundary of the $p$-regular rooted tree, that is, an infinite path, as transitive as pro- $p$ groups can. By that we mean that if $U$ is a subgroup of finite index, then the number of $U$-orbits into which the boundary of the tree decomposes grows only logarithmically with the index of $U$. To measure the transitivity of the action of a group, define the orbit growth $o_{n}(G, X)$ of a group $G$ acting transitively on a set $X$ as the maximal number of orbits of a subgroup $U$ of index at most $n$ in $G$. Orbit growth and subgroup growth are linked by the following proposition.

Proposition 5. Let $G$ be a p-group, acting transitively on a set $X$. Let $H$ be the wreath product $G \geq \mathbb{F}_{p}$ induced by this action. Then we have

$$
o_{p^{n}}(G, X) \leqslant d_{p, H}(n) \leqslant d_{H}(n) \leqslant o_{p^{n}}(G, X)+n \max _{m \leqslant n} d_{G}(m)
$$

In general, the upper bound in Proposition 5 is probably optimal. However, in special cases, the gap between the upper and the lower bound may be a lot smaller. In particular, let $G$ be a Grigorchuk group or a Gupta-Sidki group acting on the boundary of the $p$-adic tree, and let $X$ be the orbit of one boundary point, that is, one infinite branch of the tree under this action. As the action of $G$ on $X$ is a lot more transitive than the action of an arbitrary $p$-group, it may well be possible that on the right, the term $n \max _{m \leqslant n} d_{G}(m)$ could be replaced by $\mathcal{O}(n+$ $\left.\max _{m \leqslant n} d_{G}(m)\right)$. If this is true, then the lower bound $f(n) \geqslant n^{3}$ in Theorem 2 could be replaced by $f(n) \geqslant n^{2}$, and Problem 2 would be solved. 
In view of Proposition 5, the problem of constructing pro- $p$ groups of a given subgroup growth type can be reduced to the problem of finding residually finite $p$-groups acting with a given orbit growth type (as long as the orbit growth is faster than $\left.n d_{G}(m)\right)$.

THEOREM 6. Let $G$ be the Grigorchuk group or a Gupta-Sidki group. Let $f$ : $\mathbb{N} \rightarrow \mathbb{N}$ be a nondecreasing function, and assume that $\frac{f(n)}{n} \rightarrow \infty$ and that $\frac{f(n)}{p^{n}} \rightarrow$ 0 . Then there exists a transitive action of $G$ on a set $X$, such that $o_{p^{n}}(G, X) \leqslant$ $p^{4} f(n)$ for all sufficiently large $n$, and $o_{p^{n}}(G, X) \geqslant \frac{1}{p} f(n)$ for infinitely many $n$.

Theorem 2 now follows easily. Indeed, from Corollary 4, we have $d_{p, G}(n)=$ $\mathcal{O}(n)$. Thus from Proposition 5, we obtain $d_{p, H}(n)=o_{p^{n}}(G, X)+\mathcal{O}\left(n^{2}\right)$. By Theorem 6, we see that we can choose $o_{p^{n}}(G, X)$ as we want, and Proposition 3(3) then implies Theorem 2.

One of the main ingredients of our proof is the following.

THEOREM 7. Let $G$ be the Grigorchuk group or a Gupta-Sidki group acting on the p-regular rooted tree $T$. Let $X$ be the orbit under $G$ of some infinite path in $T$. Then $o_{p^{m}}(G, X) \leqslant\left(p^{5}-1\right) m+1 \leqslant p^{5} m$ for all $m$.

Here it is crucial that the number of orbits of a subgroup increases only slowly with the index. In fact, the logarithmic growth is the smallest possible and another remarkable property of these groups.

THEOREM 8. Let $G$ be a p-group acting transitively on a set $X$. Suppose $o_{n}(G$, $X)$ is unbounded. Then there exist infinitely many $m$, such that

$$
o_{p^{m}}(G, X) \geqslant(p-1) m+1 \text {. }
$$

We believe that orbit growth is of independent interest even for discrete groups. Analogous to Theorem 8, we ask the following.

PROBLEM 6. Is there a discrete group action with orbit growth less than logarithmic; in particular, is there one with orbit growth $\sim \frac{\log n}{\log \log n}$ ? Is there a slowest possible orbit growth for discrete groups?

Moreover, we wonder whether Theorem 7 is a special property of branch groups.

PROBLEM 7. Is there a class $\mathcal{V}$ of pro-p groups, such that for all $G \in \mathcal{V}$ and all transitive actions of $G$ on a set $X$ we have that $o_{p^{n}}(G, X)$ is either bounded or 
substantially larger than $n$ ? For instance, is there a class $\mathcal{V}$ such that $o_{p^{n}}(G, X)$ is either bounded or superpolynomial?

We note that the maximal orbit growth possible is linear in the index. Indeed, for any residually finite infinite group, if we take a descending chain of normal subgroups and act on the coset tree, we obtain linear growth. It is an interesting problem to find the possible orbit growth of a given group. For instance, we have the following problem.

Problem 8. Let $G$ be a p-adic analytic pro-p group. What are the possible orbit growth of actions of $G$ ?

Note that if $G$ is $p$-adic analytic and $n<o_{p^{n}}(G)<n^{2}$, then we get new types of subgroup growth. However, we do not know whether this is possible.

\section{Subgroup Growth of Self-Replicating $p$-Groups and Pro- $p$ Groups}

A group $G$ is called self-replicating if there exist an integer $k$ and a normal subgroup $G_{1} \leqslant G$ of finite index, such that $G_{1} \cong G^{(k)}$, the direct sum of $k$ copies of $G$. (We comment that this definition is different from the standard definition in the literature.) By iterating this process, we obtain $G_{n} \cong G_{n-1}^{(k)} \cong G^{\left(k^{n}\right)}$, the $n$th principal congruence subgroup of $G$. We say that $U<G$ is a congruence subgroup if $U$ contains a principal congruence subgroup. If $U$ is a congruence subgroup, then the level of $U$ is the smallest $\ell$ such that $U$ contains the $\ell$ th principal congruence subgroup. If $G$ contains a self-replicating normal subgroup of finite index and every finite index subgroup of it is a congruence subgroup, then we say that $G$ has the congruence subgroup property. (We comment that this definition is also slightly different from the standard definition for the congruence subgroup property for groups acting on trees.)

In this section, we will prove the following theorem.

THEOREM 9. Let $G$ be a finitely generated, self-replicating p-group or pro$p$ group that has the congruence subgroup property. Then $G$ has subgroup growth of type $n^{\log n}$. More precisely, suppose $G$ contains a principal congruence subgroup $N$, such that $N \cong G^{(k)},|G / N|=p^{\ell}$, and $N \leqslant \Phi_{p}(G)$. Put $d_{p}=$ $\max d_{p}(H)$, where $H$ runs over all subgroups of the finite group $G / N$. Then we have that

$$
\frac{(k-1) d_{p}(G)}{\ell} \leqslant \alpha(G) \leqslant(k-1) d_{p}(G)+d_{p} ;
$$


(2)

$$
\alpha(G)=\lim _{m \rightarrow \infty} \frac{d_{p}(m)}{m}=\sup _{m \geqslant 0} \frac{d_{p}(m)}{m+\frac{\ell}{k-1}} .
$$

Since for a $p$-group $G$ we have that $d_{p}(G)=d(\widehat{G})$ and $s_{p^{n}}(G)=s_{p^{n}}(\widehat{G})$, where $\widehat{G}$ is the pro- $p$ completion of $G$, it suffices to assume $G$ is a pro- $p$ group in the proof of the theorem. We also recall that if $G$ is a pro- $p$ group, then $\Phi_{p}(G)=$ $\Phi(G)$ and $d_{p}(G)=d(G)$.

The following is almost trivial. Nevertheless, we include the proof for completeness since direct products often show unexpected behaviour.

LEMMA 10. Let $G$ and $H$ be pro-p-groups, $m$ a natural number, and assume that there is a constant $C$, such that for all subgroups $U_{1}<G, U_{2}<H$ with $\left(G: U_{1}\right)=p^{k},\left(H: U_{2}\right)=p^{\ell}$ with $k, \ell \leqslant m$, we have $d\left(U_{1}\right) \leqslant C k+d(G)$, $d\left(U_{2}\right) \leqslant C \ell+d(H)$. Then for all subgroups $U<G \times H$ with index $p^{m}$, we have $d(U) \leqslant C m+d(G)+d(H)$.

Proof. Let $U$ be a subgroup of $G \times H$. Let $\pi: U \rightarrow H$ be the canonical projection. Then consider $U_{1}=U \cap G, U_{2}=\mathrm{im} \pi$. Take generators $g_{1}, \ldots, g_{r}$ of $U_{1}$, and generators $h_{1}, \ldots, h_{s}$ of $U_{2}$. For each $h_{i}$, choose a pre-image $\widetilde{h}_{i}$ under $\pi$. Then $g_{1}, \ldots, g_{r}, \widetilde{h}_{1}, \ldots, \widetilde{h}_{s}$ are contained in $U$, and generate a subgroup $\tilde{U}$ of $U$. We have $\tilde{U} \cap G=U \cap G$, and $\pi(\tilde{U})=\pi(U)$; thus, $(G \times H: \tilde{U})=(G \times H: U)$. Therefore, $U=\tilde{U}$, and $d(U) \leqslant r+s$.

Since $(G \times H: U)=\left(G: U_{1}\right)\left(H: U_{2}\right)$ by our assumption, we get that $r+s \leqslant \log \left(G: U_{1}\right)+d(G)+\log \left(H: U_{2}\right)+d(H)=\log (G \times H: U)+d(G)+d(H)$, and our claim follows.

LEMMA 11. Let $G$ be a finitely generated self-replicating pro-p-group that has the congruence subgroup property. Define $N, k$ and $d$ as in Theorem 9. Then a subgroup of index $p^{m}$ has at most $C m+d(G)$ generators, where $C=(k-$ 1) $d(G)+d$.

Proof. We prove our assertion by induction on $m$. If $m=0$, then $U=G$, and our claim is trivial. Now let $U$ be an open subgroup of index $p^{m}$. If $U N / N=G / N$, then in particular $U \Phi(G) / \Phi(G)=G / \Phi(G)$, and we conclude that $U=G$ again. Henceforth, we assume that $U N / N<G / N$. Put $V=U \cap N$. Then

$$
(G: U)=(G: U N)(U N: U)=(G / N: U N / N)(N: V),
$$


and thus, $(N: V)<(G: U)$. As $V \leqslant N \cong G^{(k)}$, we can use Lemma 10 and the induction hypothesis to obtain that $d(V) \leqslant C(m-1)+k d(G)$. Thus,

$$
d(U) \leqslant d(V)+d(U N / N) \leqslant d(V)+d .
$$

We deduce that

$$
\begin{aligned}
& d(U) \leqslant C(m-1)+k d(G)+d \\
& \quad=((k-1) d(G)+d)(m-1)+((k-1) d(G)+d)+d(G)=C m+d(G),
\end{aligned}
$$

and our claim is proven.

Proof of Theorem 9. We start with part (2). The inequality $\lim \frac{d(m)}{m} \leqslant \sup \frac{d(m)}{m+c}$ holds for any sequence $d(m)$ and for any positive real number $c$. For the reverse inequality, we start by showing that for any $r \geqslant 0$, we can find in $G$ a subgroup of index $p^{\ell r}$ which is isomorphic to $G^{((k-1) r+1)}$. This is done by induction on $r$. For $r=0$, we take $G$ itself. Suppose we know it for $r$; we will prove it for $r+1$. Take one of the components in the subgroup isomorphic to $G^{((k-1) r+1)}$ and replace it by $N$. The index of the new subgroup increases by $p^{\ell}$, so it is $p^{\ell(r+1)}$, while the number of components increases by $k-1$; so we obtain that the new subgroup is isomorphic to $G^{((k-1)(r+1)+1)}$ as required, and thus the induction is proved.

Let $U<G$ be a subgroup of index $p^{m}$ with $d(U)=d(m)$. By taking a subgroup isomorphic to $U$ in each component of $G^{((k-1) r+1)}$, we have that $G$ contains a subgroup $V \cong U^{((k-1) r+1)}$ of index $p^{\ell r}(G: U)^{(k-1) r+1}=$ $p^{\ell r}\left(p^{m}\right)^{(k-1) r+1}=p^{(\ell+m(k-1)) r+m}$ with $d(V)=((k-1) r+1) d(U)=$ $((k-1) r+1) d(m)$.

For a natural number $n \geqslant m$, take $r$ such that $(\ell+m(k-1)) r+m \leqslant n<$ $(\ell+m(k-1))(r+1)+m$, that is, $r=\left\lfloor\frac{n-m}{\ell+m(k-1)}\right\rfloor$; pick a subgroup $V$ as described in the last paragraph and choose $H<V$ with $(V: H)=p^{n-(\ell-m(k-1)) r-m}$. Then $(G: H)=p^{n}$, and $(V: H)<p^{\ell+m(k-1)}$; thus, $d(H) \geqslant d(V)-\ell-m(k-1)=$ $((k-1) r+1) d(m)-\ell-m(k-1)$. Note that $m, k$, and $\ell$ are constant while $n$ and $r$ tend to infinity. We conclude that

$$
\begin{aligned}
d(n) & \geqslant d(H) \geqslant((k-1) r+1) d(m)-\overbrace{\ell-m(k-1)}^{\mathcal{O}(1)} \\
& =\frac{\overbrace{(\ell+m(k-1)) r}(\ell+\mathcal{O}(1)}{m} d(m)+\mathcal{O}(1) \\
& =\frac{n-\ell r}{m} d(m)+\mathcal{O}(1) .
\end{aligned}
$$


Recall that $n=(\ell+m(k-1)) r+\mathcal{O}(1)$. Thus, $\frac{\ell r}{n}=\frac{\ell}{\ell+m(k-1)}+\mathcal{O}\left(\frac{1}{n}\right)$. Therefore, dividing the equations above by $n$, we obtain

$$
\begin{aligned}
& \frac{d(n)}{n} \geqslant \frac{n-\ell r}{n m} d(m)+\mathcal{O}\left(\frac{1}{n}\right)=\frac{d(m)}{m}\left(1-\frac{\ell}{\ell+m(k-1)}\right)+\mathcal{O}\left(\frac{1}{n}\right) \\
& =\frac{d(m)}{m} \frac{m(k-1)}{\ell+m(k-1)}+\mathcal{O}\left(\frac{1}{n}\right)=\frac{d(m)}{\frac{\ell}{k-1}+m}+\mathcal{O}\left(\frac{1}{n}\right),
\end{aligned}
$$

and our claim follows.

Finally note that the lower bound in Theorem 9(1) follows from Theorem 9(2) since

$$
\sup _{m \geqslant 0} \frac{d(m)}{m+(\ell /(k-1))} \geqslant \frac{d(0)}{\frac{\ell}{k-1}}=\frac{(k-1) d(G)}{\ell} .
$$

The most notable examples of self-replicating $p$-groups are the Grigorchuk group and the Gupta-Sidki groups. Therefore, we can prove Corollary 4.

Proof of Corollary 4. Suppose $G$ is the Grigorchuk group. Since $G$ is a 2-group, its profinite completion is the same as its pro- 2 completion. It follows from [5, page 167, Proposition 8] that $G$ has a self-replicating subgroup $K$ of index 16, which is 3-generated. Write $K_{i}$ for the $i$ th principal congruence subgroup of $K$. Then $K_{1}$ is of index 4 with $K_{1} \cong K \times K$, and $K / K_{1} \cong C_{4}$. Also, from [5, page 169, Proposition 10], $G$ has the congruence subgroup property. Thus, we can assume that $G$ is a pro-2 group with the congruence subgroup property. Then $\Phi_{p}(K)$ contains $N=K_{2} \cong K_{1} \times K_{1} \cong K^{(4)}$ of index $4^{3}=2^{6}$ in $K$. Hence, in the theorem, we have $\ell=6, k=4$ and $d=3$; thus, $\frac{3}{2} \leqslant \alpha(K) \leqslant 12$. From Proposition 3, we obtain that the same inequality holds for $\alpha(G)$, and our claim follows.

Suppose $G$ is the Gupta-Sidki $p$-group. Since $G$ is a $p$-group, its profinite completion is the same as its pro- $p$ completion. The lower bound for the GuptaSidki groups is just the lower bound for non- $p$-adic analytic pro- $p$ groups, established by Shalev [14]. Note that the following properties of the Gupta-Sidki groups were established by Sidki [13] for $p=3$. These can be generalized easily for $p>3$; see Garrido [4, Section 2] for proofs of most of these generalizations. For the upper bound, we use the fact that $K$, the commutator of subgroup of $G$, is self-replicating, and we have that $\Phi_{p}(K) \geqslant K_{2}, K_{1} \cong K^{(p)},\left(K: K_{2}\right)=p^{p^{2}-1}$, and $d(K)=p-1$. Moreover, $K$ has the congruence subgroup property, and $d=p(p-1)$, the maximum being attained by the subgroup $K_{1} / K_{2}$ of $K / K_{2}$. Hence, by Theorem 9, we have $\alpha(G) \leqslant(k-1) d(K)+d=3 p^{2}-4 p+1$, and our claim follows from Proposition 3. 


\section{Orbit growth and subgroup growth}

For the proof of Proposition 5, we need the following results.

LeMmA 12. Let $G$ be an m-generated $p$-group and $M$ a d-generated $\mathbb{F}_{p} G$ module. Let $N$ be a submodule of $M$, which as an $\mathbb{F}_{p}$-vector space has codimension 1. Then $N$ is at most $(d+m-1)$-generated as an $\mathbb{F}_{p} G$-module.

Proof. Let $g_{1}, \ldots, g_{m}$ be generators of $G$ and $v_{1}, \ldots, v_{d}$ be generators of $M$ as an $\mathbb{F}_{p} G$-module. Then $\left\{v_{i}^{g} \mid i \leqslant d, g \in G\right\}$ generates $M$ as an $\mathbb{F}_{p}$-vector space. Let $\varphi: M \rightarrow \mathbb{F}_{p}$ be the module homomorphism given by the canonical map $M \rightarrow M / N$. Note that $\operatorname{ker} \varphi=N$. Since the action of a $p$-group on a cyclic group of order $p-1$ is trivial, we have that $\mathbb{F}_{p}$ is a trivial $\mathbb{F}_{p} G$-module, that is, for all $m \in M$, we obtain $\varphi\left(m^{g}\right)=\varphi(m)^{g}=\varphi(m)$.

Suppose without loss of generality that $\varphi\left(v_{1}\right) \neq 0$. We claim that as a vector space $N$ is generated by

$$
D=\left\{\varphi\left(v_{i}\right) v_{1}^{g}-\varphi\left(v_{1}\right) v_{i}^{h} \mid 1 \leqslant i \leqslant d, g, h \in G\right\} .
$$

As $\varphi\left(\varphi\left(v_{i}\right) v_{1}^{g}-\varphi\left(v_{1}\right) v_{i}^{h}\right)=0$, we have that $D \subseteq N$. Let $m \in N$ be an arbitrary element. Write $m=\sum_{i, j} \lambda_{i j} v_{i}^{h_{j}}$, where $h_{j} \in G$. We have that

$$
\sum_{i, j} \lambda_{i j} v_{i}^{h_{j}}+\sum_{i, j} \frac{\lambda_{i j}}{\varphi\left(v_{1}\right)} \underbrace{\left(\varphi\left(v_{i}\right) v_{1}-\varphi\left(v_{1}\right) v_{i}^{h_{j}}\right)}_{\in D}=\left(\sum_{i, j} \frac{\lambda_{i j}}{\varphi\left(v_{1}\right)} \varphi\left(v_{i}\right)\right) v_{1} .
$$

The left-hand side is in $N$, and since $v_{1}$ is not in $N$, the right-hand side can only be in $N$ if it vanishes. But then we have represented $m$ as a linear combination of elements of $D$, that is, $D$ generates $N$ as a vector space.

We next claim that as an $\mathbb{F}_{p} G$-module $N$ is generated by the set

$$
\left\{v_{1}-v_{1}^{g_{1}}, v_{1}-v_{1}^{g_{2}}, \ldots, v_{1}-v_{1}^{g_{m}}, \varphi\left(v_{i}\right) v_{1}-\varphi\left(v_{1}\right) v_{2}, \ldots, \varphi\left(v_{i}\right) v_{1}-\varphi\left(v_{1}\right) v_{d}\right\} .
$$

Let $V$ be the $\mathbb{F}_{p} G$-module generated by this set. It suffices to show that $D \subseteq V$. We show first that $v_{1}-v_{1}^{g} \in V$ for all $g \in G$. To do so, we write $g$ as a word in $\left\{g_{1}^{ \pm 1}, \ldots, g_{m}^{ \pm 1}\right\}$, say $g=g_{i_{1}}^{\epsilon_{1}} \ldots g_{i_{k}}^{\epsilon_{k}}$. Then

$$
v_{1}-v_{1}^{g}=v_{1}-v_{1}^{g_{i_{k}}^{\epsilon_{k}}}+\left(v_{1}-v_{1}^{g_{i_{k-1}}^{\epsilon_{k-1}}}\right)^{g_{i_{k}}^{\epsilon_{k}}}+\cdots+\left(v_{1}-v_{1}^{g_{i_{1}}^{\epsilon_{1}}}\right)^{g_{i_{2}}^{\epsilon_{2}} \ldots g_{i_{k}}^{\epsilon_{k}}},
$$

and since $v_{1}-v_{1}^{g^{-1}}=-\left(v_{1}-v_{1}^{g}\right)^{g^{-1}}$, we find that $v_{1}-v_{1}^{g} \in V$ for all $g \in G$.

Next for $g, h \in G$, we have $\varphi\left(v_{i}\right) v_{1}^{g}-\varphi\left(v_{1}\right) v_{i}^{h} \in V$ since

$$
\varphi\left(v_{i}\right) v_{1}^{g}-\varphi\left(v_{1}\right) v_{i}^{h}=\varphi\left(v_{i}\right)\left(v_{1}-v_{1}^{h g^{-1}}\right)^{g}+\left(\varphi\left(v_{i}\right) v_{1}-\varphi\left(v_{1}\right) v_{i}\right)^{h} .
$$


We have found a generating system consisting of $d+m-1$ elements, and our claim follows.

Proof of Proposition 5. We first give the upper bound. Let $U$ be a subgroup of $H$ of index $p^{n}$, let $B$ be the base group of the wreath product, and denote by $\pi$ the canonical projection $\pi: H \rightarrow G$. Let $O_{1}, \ldots, O_{N}$ be a complete list of the orbits of $\pi(U)$. For each orbit, pick an element $x_{i} \in O_{i}$ and define $b_{i} \in B$ to have $x_{i}$ coordinate 1 and all other coordinates 0 . Then $b_{1}, \ldots, b_{N}$ generate $B$ as a $\pi(U)$-module. We conclude that $\pi^{-1}(\pi(U)) \geqslant U$ is generated by

$$
N+d(\pi(U)) \leqslant N+\max _{m \leqslant n} d_{G}(m)
$$

elements.

Since $B$ is Abelian, we observe that the action of $U$ on $B$ factorizes through $\pi(U)$. Therefore, we need to bound the number of generators of $U \cap B$ as a $\pi(U)$-module. As $\pi(U)$ is a $p$-group and $U \cap B$ is of finite index in $B$, there exists a sequence of $\pi(U)$-submodules $B=M_{0}>M_{1}>\cdots>M_{\ell}=U \cap B$ with $\left(M_{j}: M_{j+1}\right)=p$. We can repeatedly apply Lemma 12 to find that for each $1 \leqslant j \leqslant \ell$, we have that $M_{j}$ can be generated by $\leqslant N+j(d(\pi(U))-$ $1) \leqslant N+j \max _{m \leqslant n} d_{G}(m)$ elements. Hence, $U$ can be generated by $\leqslant N+$ $n \max _{m \leqslant n} d_{G}(m)$ elements.

For the lower bound, define a map $\varphi_{i}: U \rightarrow \mathbb{F}_{p}, 1 \leqslant i \leqslant N$, as follows. Write an element of $U$ as $(g, f)$, where $g \in G$ and $f: X \rightarrow \mathbb{F}_{p}$ has finite support. Then we define $\varphi_{i}: U \rightarrow \mathbb{F}_{p}$ by $\varphi_{i}((g, f))=\sum_{x \in O_{i}} f(x)$. This map is a homomorphism since

$$
\begin{aligned}
\varphi_{i}((g, f)(\tilde{g}, \tilde{f}))= & \varphi_{i}\left(\left(g \tilde{g}, f^{\tilde{g}}+\tilde{f}\right)\right)=\sum_{x \in O_{i}} f^{\tilde{g}}(x)+\sum_{x \in O_{i}} \tilde{f}(x) \\
= & \sum_{x \in O_{i}} f\left(x^{\tilde{g}}\right)+\sum_{x \in O_{i}} \tilde{f}(x)=\sum_{x \in O_{i}} f(x) \\
& +\sum_{x \in O_{i}} \tilde{f}(x)=\varphi_{i}((g, f))+\varphi_{i}((\tilde{g}, \tilde{f})) .
\end{aligned}
$$

Let $V=\mathbb{F}_{p}^{N}$; we define $\varphi: U \rightarrow V$ by $\varphi((g, f))=\left(\varphi_{i}((g, f))\right)_{i=1}^{N}$. For $i$ in the range $1 \leqslant i \leqslant N$, fix an element $x_{i} \in O_{i}$. Given $\left(a_{i}\right) \in \mathbb{F}_{p}^{N}$, we define $f: X \rightarrow \mathbb{F}_{p}$ by $f(x)=a_{i}$ if $x=x_{i}$ for some $i \leqslant N$, and $f(x)=0$ otherwise. Then $\varphi(1, f)=\left(a_{i}\right)$. Thus, $\varphi$ is a surjective homomorphism.

We conclude that if $U$ is a subgroup of $G$, then $\pi^{-1}(U)$ maps onto $\mathbb{F}_{p}^{N}$. By the definition of orbit growth, there exists a subgroup $U$ of index $p^{n}$, which acts with $o_{n}(G, X)$ orbits, and $(G: U)=\left(H: \pi^{-1}(U)\right)$; thus $\pi^{-1}(U)$ has index $p^{n}$ and maps onto $\mathbb{F}_{p}^{o_{n}(G)}$. Since $d_{p}(U)$ equals the maximal dimension of an $\mathbb{F}_{p}$-vector 
space onto which $U$ surjects, we obtain $o_{n}(G) \leqslant d_{p, H}(n)$. Finally, the inequality $d_{p, H}(n) \leqslant d_{H}(n)$ always holds, and the proof is complete.

Next we prove Theorem 8 .

Proof of Theorem 8. Pick a natural number $n$. Let $U$ be a finite index subgroup, which acts with at least $n$ orbits on $X$. Pick a normal subgroup $N \triangleleft G$ of finite index within $U$. Write $\mathcal{O}$ for the set of orbits of $N$ acting on $X$. We have $|\mathcal{O}| \geqslant n$, as $N$ has at least as many orbits as $U$. Clearly, $G / N$ acts transitively on $\mathcal{O}$. Choose $o \in \mathcal{O}$, and let $H$ be the pre-image in $G$ of the stabilizer $(G / N)_{o}$. We will show that $H$ has at least $(p-1) \log (G: H)+1$ orbits.

Note that the orbits of $(G / N)_{o}$ on $\mathcal{O}$ are in one-to-one correspondence with the orbits of $H$ on $X$. For instance, if $\left\{o_{1}, o_{2}, \ldots, o_{r}\right\}$ is an orbit of $(G / N)_{o}$ on $\mathcal{O}$, then the corresponding orbit of $H$ on $X$ is $\cup_{i} o_{i}$. In particular, the number of orbits of $H$ on $X$ equals the number of orbits of $(G / N)_{o}$ on $\mathcal{O}$. Let $\mathcal{O}_{1}, \ldots$, $\mathcal{O}_{k}$ be the orbits of $(G / N)_{o}$, and put $(G: H)=\left(G / N:(G / N)_{o}\right)=p^{m}$. We have $p^{m}=|\mathcal{O}| \geqslant n$; in particular, by choosing $n$ appropriately, we can make $m$ arbitrarily large. Since $G / N$ is a $p$-group, we have for each $i$ that $\left|\mathcal{O}_{i}\right|=p^{m_{i}}$ for some $m_{i} \geqslant 0$. We conclude that $\sum_{i=1}^{k} p^{m_{i}}=p^{m}$. Note that at least one of the $m_{i}$ equals 0 , since $(G / N)_{o}$ has the fixed point $o$, say $m_{1}=0$.

We claim that every integral solution of the equation $\sum_{i=1}^{k} p^{m_{i}}=p^{m}$ with $m_{1}=0$ satisfies $k \geqslant(p-1) m+1$. We prove our claim by induction over $m$. For $m=1$, the only integral solution is $1+1+\cdots+1=p$. Now assume our claim holds for $m-1$. Let $\mu_{j}$ be the number of indices $i$ with $m_{i}=j$. Then we have $\sum_{j} \mu_{j} p^{j}=p^{m}$. We have to show that $\mu_{0} \geqslant 1$ implies $\sum \mu_{j} \geqslant(p-1) m+1$. Let $\left(\mu_{0}, \ldots, \mu_{m}\right)$ be a tuple minimizing $\sum \mu_{j}$. Then

$$
\mu_{0} \equiv \sum_{j} \mu_{j} p^{j}=p^{m} \equiv 0 \quad(\bmod p)
$$

together with $\mu_{0} \geqslant 1$ we conclude $\mu_{0} \geqslant p$. If $\mu_{0} \geqslant 2 p$, then the tuple $\left(\mu_{0}-p\right.$, $\left.\mu_{1}+1, \ldots, \mu_{k}\right)$ is also a solution of $\sum_{j} \mu_{j} p^{j}=p^{m}$. The sum of the entries of the new tuple is $\left(\sum \mu_{j}\right)-(p-1)$, contradicting minimality. Hence, we may assume $\mu_{0}=p$. We now get $1+\sum_{j \geqslant 1} \mu_{j} p^{j-1}=p^{m-1}$; thus, by the inductive hypothesis, we conclude $\sum_{j \geqslant 1} \mu_{j} \geqslant(p-1)(m-1)$. Together with $\mu_{0}=p$ we obtain $\sum_{j \geqslant 0} \mu_{j} \geqslant(p-1) m+1$, as claimed.

\section{Proofs of Theorems 1 and 7}

For $p=2$, let $G$ be the first Grigorchuk group and let $K$ be the self-replicating subgroup of index 16 in it, while for $p>2$, let $G$ be the Gupta-Sidki $p$-group and let $K$ be the self-replicating subgroup of $G$ of index $p^{2}$ (see [4]). Let us 
emphasize that in this section, we take $G$ and $K$ to be the discrete $p$-groups rather than their pro- $p$ completions.

Pervova in [9] showed that $G$ has no maximal subgroups of infinite index; thus $\Phi_{p}(G)=\Phi(G)$, the Frattini subgroup of $G$, and $d_{p}(G)=d(G)$. This was generalized to finite index subgroups of the Grigorchuk groups and the GuptaSidki groups by Grigorchuk and Wilson [6], and to finite index subgroups of multiedge spinal torsion group by Alexoudas, Klopsch and Thillaisundaram in [1]. We can therefore work with the discrete $p$-groups and later pass to their pro$p$ completion without changing the results of our computation. The advantage of doing so is that for discrete groups, wreath products are much easier to analyse than for topological groups.

Let $T$ be the $p$-regular rooted tree; $G$ acts naturally on $T$ and so does $K$. Let $T_{n}$ be the $n$th level of the tree and let $S t(n)=S t_{G}\left(T_{n}\right)$ be the stabilizer of $T_{n}$, where the root is considered as $T_{0}$; thus, $\operatorname{St}(0)=G$. Note that $\operatorname{St}(1)$ contains $K^{(p)}$ geometrically, that is, each component $K$ acts independently on the corresponding child tree of the first level. For $p=2$ this was proven by Grigorchuk [5, page 167, Proposition 8], for $p=3$ by Sidki [13], and for $p>3$ by Fernandez-Alcober and Zugadi-Reizabal [3].

Clearly, $K$ acts on the set of infinite paths in $T$. Pick one such path $x$, and let $X=\left\{x^{g}: g \in K\right\}$ be the orbit of $x$ under $K$.

Proof of Theorem 7. It is easier to work with $K$ in place of $G$. As $(G: U) \geqslant$ ( $K: K \cap U$ ), it is enough to consider the case when $U$ is a subgroup of $K$. We will show by induction on $m$ that if $U$ is a subgroup of $K$ with $(K: U)=p^{m}$, then $U$ has at most $\left(p^{5}-1\right) m+1$ orbits on $X$.

For $m=0$, we have to show that $K$ acts transitively on $X$. Pick $g \in G$. We have to show that there exists some $k \in K$ such that $x^{g}=x^{k}$. As $K$ acts transitively on each level, there exists for every $i$ some $k_{i}$, such that $x^{g}$ and $x^{k_{i}}$ coincide on the first $i$ nodes. We know that $K$ is closed in $G$ with respect to the topology induced by $T$, and that $K$ is compact. Hence, we can choose a subsequence $k_{i_{j}}$ converging to some element $k \in K$, which satisfies $x^{g}=x^{k}$.

Henceforth, we assume $m \geqslant 1$. We have that $\Phi_{p}(K)$ contains $S t$ (5). For $p=2$ this was shown by Grigorchuk [5, page 168, Proposition 9]; for $p \geqslant 3$ Garrido [4, Proposition 2.6] actually proved $\Phi_{p}(K) \geqslant S t(4)$. Since $K$ is a $p$-group and $U$ is a proper subgroup of $K$ of finite index, we have $U \Phi_{p}(K) \neq K$ and hence $U S t(5) \neq K$. Thus, we have

$$
(\operatorname{St}(5): U \cap S t(5))=(U S t(5): U)<(K: U) .
$$

As $\operatorname{St}(5)$ contains $K^{\left(p^{5}\right)}$ geometrically, we have that

$$
\left(K^{\left(p^{5}\right)}: U \cap K^{\left(p^{5}\right)}\right) \leqslant(S t(5): U \cap S t(5))<(K: U) .
$$


Because $U \cap K^{\left(p^{5}\right)}$ is a subgroup of $U$, the number of orbits of $U$ on $X$ is bounded from above by the number of orbits of $U \cap K^{\left(p^{5}\right)}$.

For $1 \leqslant i \leqslant p^{5}$, let $X_{i}$ be the subset of $X$ consisting of the paths passing through the $i$ th vertex of the fifth level. Then $X$ is a disjoint union of the $X_{i}$ and each $X_{i}$ is invariant under $U \cap K^{\left(p^{5}\right)}$ because $U \cap K^{\left(p^{5}\right)} \leqslant S t(5)$. Thus, the number of orbits of $U \cap K^{\left(p^{5}\right)}$ acting on $X$ is the sum of the number of orbits of $U \cap K^{\left(p^{5}\right)}$ acting on $X_{i}$. Write $K^{\left(p^{5}\right)}=K_{1} \times \cdots \times K_{p^{5}}$, where $K_{i} \cong K$. Let $\widetilde{U}_{i}$ be the projection of $U \cap K^{\left(p^{5}\right)}$ onto $K_{i}$. The action of $U \cap K^{\left(p^{5}\right)}$ on $X_{i}$ factors through $\widetilde{U}_{i}$, so the number of orbits of $U \cap K^{\left(p^{5}\right)}$ acting on $X_{i}$ is the number of orbits of $\widetilde{U}_{i}$ acting on each $X_{i}$.

For $1 \leqslant i \leqslant p^{5}$, let $U_{i}$ be the projection of $U \cap\left(K_{1} \times \cdots \times K_{i}\right)$ onto $K_{i}$. Then $U_{i}$ is a subgroup of $\widetilde{U}_{i}$, so the number of orbits of $\widetilde{U}_{i}$ acting on $X_{i}$ is bounded above by the number of orbits of $U_{i}$ acting on $X_{i}$.

Using induction on $r$, it is easy to prove that $\left(K_{1} \times \cdots \times K_{r}: U \cap\left(K_{1} \times \cdots \times\right.\right.$ $\left.\left.K_{r}\right)\right)=\prod_{i=1}^{r}\left(K_{i}: U_{i}\right)$. We deduce that

$$
\prod_{i=1}^{p^{5}}\left(K_{i}: U_{i}\right)=\left(K^{\left(p^{5}\right)}: U \cap K^{\left(p^{5}\right)}\right)=\left(U K^{\left(p^{5}\right)}: U\right) \leqslant(U \Phi(K): U)<(K: U) ;
$$

in particular, each single factor on the left is strictly smaller than $p^{m}$. Viewing $U_{i}$ as a subgroup of $K_{i}$ acting on the $i$ th subtree, we can apply our induction hypothesis to find that the number of orbits of $U \cap K^{\left(p^{5}\right)}$ acting on $X$ is at most

$$
\begin{aligned}
\sum_{i=1}^{p^{5}}\left(\left(p^{5}-1\right) \log \left(K_{i}: U_{i}\right)+1\right) & =\left(p^{5}-1\right) \log \left(K^{\left(p^{5}\right)}: U \cap K^{\left(p^{5}\right)}\right)+p^{5} \\
& \leqslant\left(p^{5}-1\right)(m-1)+p^{5}=\left(p^{5}-1\right) m+1
\end{aligned}
$$

and the proof is complete.

By taking componentwise action, we obtain for $k \geqslant 2$ a transitive permutation group $\left(K^{(k)}, X^{(k)}\right)$, where $\left(x_{1}, \ldots, x_{k}\right)^{\left(g_{1}, \ldots, g_{k}\right)}=\left(x_{1}^{g_{1}}, \ldots, x_{k}^{g_{k}}\right)$. For a set $Y$, we write $\mathbb{F}_{p}^{(Y)}$ for the direct sum of $\mathbb{F}_{p}$ indexed by $Y$. Let $H_{k}$ be the restricted wreath product $\mathbb{F}_{p} 2\left(K^{(k)}, X^{(k)}\right) \cong \mathbb{F}_{p}^{\left(X^{(k)}\right)} \rtimes K^{(k)}$. Then $H_{k}$ is a finitely generated $p$ group. Let $B$ be the base group of the wreath product and $\pi: H_{k} \rightarrow K^{(k)}$ be the projection onto the active group.

In general, wreath products of residually finite groups need not be residually finite. However, in all situations we are interested in, residually finiteness can be obtained from the following. 
LEMMA 13. Let $\Gamma$ be a residually finite group and $\Omega$ a set on which $\Gamma$ acts transitively. Suppose that for all $x, y \in \Omega$, there exists a finite index subgroup $\Delta$ such that $x$ and $y$ are not in the same $\Delta$-orbit. Then the restricted wreath product $\mathbb{F}_{p}<\Gamma$ given by the action is residually finite.

Proof. Let $(f, \gamma)$ be a nontrivial element of the wreath product, where $f: \Omega \rightarrow$ $\mathbb{F}_{p}$ is a function with a finite support and $\gamma \in \Gamma$. If $\gamma \neq 1$, then there exists a subgroup $\Delta<\Gamma$ of finite index not containing $\gamma$, and the pre-image of $\Delta$ under the canonical projection is a finite index subgroup not containing $(f, \gamma)$. Now suppose that $\gamma=1, f$ does not vanish identically, and has support supp $f=\left\{x_{1}\right.$, $\left.\ldots, x_{n}\right\} \neq \emptyset$. For $1 \leqslant i<j \leqslant n$, choose a finite index subgroup $\Delta_{i j}$ such that $x_{i}$ and $x_{j}$ are not in the same $\Delta_{i j}$-orbit. Then $\Delta=\bigcap \Delta_{i j}$ is a finite index subgroup, which acts on $\Omega$ with finitely many orbits $\Omega_{1}, \ldots, \Omega_{N}$. Then

$$
M=\left\{m: \Omega \rightarrow \mathbb{F}_{p}:|\operatorname{supp} m|<\infty, \forall i \leqslant N: \sum_{x \in \Omega_{i}} m(x)=0\right\}
$$

is $\Delta$-invariant and of finite index in $\mathbb{F}_{p}^{(\Omega)}$. Hence, $\left\{(m, \delta) \in \mathbb{F}_{p} 2 \Gamma: m \in M\right.$, $\delta \in \Delta\}$ is a finite index subgroup of $\Gamma$, which does not contain $(f, \gamma)$, because each $\Omega_{i}$ contains at most one point on which $f$ does not vanish, and $f$ does not vanish identically.

COROLlARY 14. $H_{k}$ is residually finite.

Proof. Let $x, y \in X$ be different paths. In view of Lemma 13, we have to show that there exists a finite index subgroup $U$ of $G$, such that $x$ and $y$ are in different orbits of $U$. But if $x$ and $y$ are different, there is some $\ell$ such that their intersection with the $\ell$ th level is different, and $S t(\ell)$ does what we need.

We now show that there are positive constants $c_{1}, c_{2}$, such that $c_{1} n^{k} \leqslant$ $o_{p^{n}}\left(G^{(k)}, X^{(k)}\right) \leqslant c_{2} n^{k}$. We start with a lower bound.

LEMMA 15. Let $\Gamma$ be a p-group acting on $T$. Then for every natural number $\ell$, we have that $\left(\Gamma: S t_{\Gamma}(\ell)\right) \leqslant p^{p^{\ell}}$.

Proof. We know there is an injective homomorphism $\Gamma / S t_{\Gamma}(\ell) \rightarrow S_{p^{\ell}}$. The image of this homomorphism is a $p$-subgroup and hence a subgroup of the $p$ Sylow subgroup of $S_{p^{\ell}}$. The latter has order $p^{p^{\ell}-1}$, and our claim follows.

LEMMA 16. Let $G$ be the Grigorchuk group or a Gupta-Sidki group. We have that $\left(G^{(k)}: S t(\ell)^{(k)}\right) \leqslant p^{k p^{\ell}}$, and $\operatorname{St}(\ell)^{(k)}$ has at least $p^{k \ell}$ orbits on $X^{(k)}$. 
In particular,

$$
o_{p^{n}}\left(G^{(k)}, X^{(k)}\right) \geqslant \frac{1}{(p k)^{k}} n^{k}
$$

Proof. The bound for the index follows immediately from Lemma 15 . Now recall that $G^{(k)}$ acts transitively on $T_{\ell}^{(k)}$, whereas $S t(\ell)^{(k)}$ acts trivially on $T_{\ell}^{(k)}$. Thus, if $\left(x_{1}, \ldots, x_{k}\right),\left(x_{1}^{\prime}, \ldots, x_{k}^{\prime}\right) \in X^{(k)}$ are in the same $S t(\ell)^{(k)}$-orbit, we have for all $i$ that $x_{i}$ and $x_{i}^{\prime}$ pass through the same point in level $\ell$. Therefore, the number of orbits is at least the number of $k$-tuples of vertices of level $\ell$, which is $\left(p^{\ell}\right)^{k}=p^{k \ell}$. We conclude that

$$
o_{p^{n}}\left(G^{(k)}, X^{(k)}\right) \geqslant \max _{\left\{\ell: k p^{\ell} \leqslant n\right\}} p^{k \ell} \geqslant p^{k(\log (n / k)-1)}=\frac{1}{(p k)^{k}} p^{k \log n}=\frac{1}{(p k)^{k}} n^{k},
$$

proving the last claim of the lemma.

One can give more precise bounds for the index of $S t(\ell)$. Doing so is essentially equivalent to determining the Hausdorff dimension of $G$, which was done for $p=2$ by Grigorchuk [5, Section 5], and for $p \geqslant 3$ in vast generality by Zugadi-Reizabal [15].

Now we turn to upper bounds for $o_{p^{n}}\left(G^{(k)}, X^{(k)}\right)$.

Lemma 17. Let $(\Gamma, \Omega),(\Delta, \Lambda)$ be two permutation groups. Let $U$ be a subgroup of $\Gamma \times \Delta$. Suppose that $U \cap \Gamma$ has $m$ orbits on $\Omega$, and $U \Gamma / \Gamma<\Delta$ has $n$ orbits on $\Lambda$. Then $U$ has at most mn orbits on $\Omega \times \Lambda$.

Proof. Let $x_{1}, \ldots, x_{m}$ be representatives of the orbits of $U \cap \Gamma$ acting on $\Omega$, and $y_{1}, \ldots, y_{n}$ be representatives of $U \Gamma / \Gamma$ acting on $\Lambda$. Then by considering the action of $U \Gamma / \Gamma$ on $\Lambda$, every element $(x, y) \in \Omega \times \Lambda$ is equivalent to an element of the form $\left(z, y_{j}\right), 1 \leqslant j \leqslant n$. Take an element $u \in U \cap \Gamma$ such that $z^{u}=x_{i}$ for some $1 \leqslant i \leqslant m$. By applying it to $\left(z, y_{j}\right)$, we obtain that $(x, y)$ is equivalent $\left(x_{i}, y_{j}\right)$.

LEMMA 18. Let $U$ be a subgroup of $K^{(k)}$ of index $p^{n}$. Then $U$ has at most $\mathcal{O}\left(n^{k}\right)$ orbits on $X^{(k)}$.

Proof. We prove our claim by induction on $k$. The case $k=1$ is Theorem 7 . Now suppose that $k \geqslant 2$, and our claim is already shown for all smaller values of $k$. Write $K^{(k)}=K_{1} \times \cdots \times K_{k}$. Assume that $\left(U K_{1}: U\right)=\left(K_{1}: U \cap K_{1}\right)=p^{m}$. Then from the induction hypothesis for $k=1$, we deduce that $U \cap K_{1}$ acts with $\mathcal{O}(m)$ orbits on $X$. Note that $U K_{1} / K_{1}$ acts on $X^{(k)} / K_{1} \cong X^{(k-1)}$, and that $\left(K^{(k)} / K_{1}\right.$ : $\left.U K_{1} / K_{1}\right)=\left(K^{(k)}: U K_{1}\right)=\frac{\left(K^{(k)}: U\right)}{\left(U K_{1}: U\right)}=p^{n-m}$; applying the induction hypothesis 
for $k-1$, we find that the number of orbits of this action is $\mathcal{O}\left((n-m)^{k-1}\right)$. Lemma 17 implies that $U$ itself has $\mathcal{O}\left(m(n-m)^{k-1}\right)=\mathcal{O}\left(n^{k}\right)$ orbits, and the proof of the general case is also complete.

Proof of Theorem 1. For $k=1$, this is Corollary 4. From Lemmas 16 and 18, we obtain

$$
c_{1} n^{k} \leqslant o_{p^{n}}\left(G^{(k)}, X^{(k)}\right) \leqslant c_{2} n^{k}
$$

for some positive constants $c_{1}$ and $c_{2}$. Theorem 9 implies $d_{G^{(k)}}(n) \leqslant C n$. Applying Proposition 5 with these values, we obtain $c_{1} n^{k} \leqslant d_{p, H_{k}}(n) \leqslant c_{2} n^{k}+$ $C n^{2}$, which for $k \geqslant 2$ implies our claim.

\section{Trees with a single infinite path}

In this section, we consider subtrees $S \subseteq T$, which contain exactly one infinite path. We denote by $\operatorname{Inj}(S, T)$ the set of injective root preserving graph homomorphisms $\varphi: S \rightarrow T$. The action of $G$ on the nodes of $T$ induces an action of $G$ on $\operatorname{Inj}(S, T)$ via $\varphi^{g}(x)=\varphi(x)^{g}$. As usual, $\varphi^{G} \subseteq \operatorname{Inj}(S, T)$ denotes the orbit containing $\varphi$ under this action.

The following theorem implies Theorem 6.

THEOREM 19. Let $f: \mathbb{N} \rightarrow \mathbb{N}$ be a function, such that $\frac{f(n)}{n} \rightarrow \infty$ and $\frac{f(n)}{p^{n}} \rightarrow 0$. Then there exist a tree $S \subseteq T$, which has a single infinite path, and a map $\varphi \in$ $\operatorname{Inj}(S, T)$, such that for all $n$ large enough and for all $U<G$ with $(G: U)=p^{n}$, we have that $U$ acts with at most $p^{4} f(n)$ orbits on $\varphi^{G}$, and there exist infinitely many $n$ and subgroups $U$ with $(G: U)=p^{n}$, such that $U$ acts with at least $\frac{1}{p} f(n)$ orbits on $\varphi^{G}$.

Before proving the theorem, we need to study stabilizers of antichains in $T$. The subgroups determining the lower bound of the orbit growth in the theorem will be certain stabilizers of antichains in $T$. If $\mathcal{A}=\left\{x_{1}, \ldots, x_{k}\right\}$ is a maximal antichain in $T$, then we denote by $T^{\mathcal{A}}$ the finite tree obtained from $T$ by deleting all vertices below any element of $\mathcal{A}$. Denote by $G_{\mathcal{A}}$ the pointwise stabilizer of $\mathcal{A}$ in $T$. If $S$ is a subtree of $T$, we put $S^{\mathcal{A}}=S \cap T^{\mathcal{A}}$.

We say that a subtree $S$ is complete, if $v \in S$ is a vertex and if $w$ is a sibling vertex of $v$, then $w \in S$ as well. The completion $\bar{S}$ of $S$ is the smallest complete subtree of $T$ containing $S$.

LEMMA 20. Let $S$ be a subtree of $T$, and denote by $\bar{S}$ the completion of $S$ in $T$. Suppose that $\bar{\varphi} \in \operatorname{Inj}(\bar{S}, T)$ is an extension of $\varphi \in \operatorname{Inj}(S, T)$.

(1) The restriction $\rho: \operatorname{Inj}(\bar{S}, T) \rightarrow \operatorname{Inj}(S, T), \rho(\bar{\psi})=\left.\bar{\psi}\right|_{S}$ defines a bijection between $\bar{\varphi}^{G}$ and $\varphi^{G}$. 
(2) Let $U$ be a subgroup of $G$. Then $\rho$ induces a bijection between the $U$-orbits on $\bar{\varphi}^{G}$ and the $U$-orbits on $\varphi^{G}$.

Proof. Note that the $G$-action and $\rho$ commute. Thus, $\rho\left(\bar{\varphi}^{g}\right)=\rho(\bar{\varphi})^{g}=\varphi^{g}$ for all $g \in G$, so $\rho$ is surjective on $\varphi^{G}$. Suppose $\rho\left(\bar{\varphi}^{g}\right)=\rho(\bar{\varphi})$. Each $y \in \bar{S}$ has a sibling $x \in S$. Since $\rho\left(\bar{\varphi}^{g}\right)=\rho(\bar{\varphi})$, we have that $\bar{\varphi}(x)^{g}=\bar{\varphi}^{g}(x)=\bar{\varphi}(x)$. Now $\langle g\rangle$ acts on $\bar{\varphi}(x)$ and its siblings. As $G$ is a $p$-group, $\langle g\rangle$ is a finite $p$-group. Since $\bar{\varphi}(x)$ and its siblings form a set of size $p$ and $g$ fixes one element, we obtain that $\langle g\rangle$ acts trivially on it. Thus, $\bar{\varphi}^{g}(y)=\bar{\varphi}(y)^{g}=\bar{\varphi}(y)$, as $\bar{\varphi}(y)$ is a sibling of $\bar{\varphi}(x)$. We conclude that $\bar{\varphi}^{g}=\bar{\varphi}$, so $\rho$ is injective. This completes the proof of part (1). As $\rho$ commutes with the action of $G$, part (2) follows trivially.

LEMMA 21. Let $G$ be the Grigorchuk or a Gupta-Sidki group. Let $v$ be a vertex of level $\ell$. Let $\rho$ be the restriction of the action of $G$ on $T$ to $T_{v}$, the tree with root $v$. Then $\rho\left(G_{v}\right) \cong G$ as a permutation group and $\left(\rho\left(G_{v}\right): \rho(S t(\ell)) \leqslant p^{4}\right.$.

Proof. We first prove that $\rho\left(G_{v}\right) \cong G$ as a permutation group. Suppose first that $\ell=1$. We adopt the notation from [5], where for automorphisms $\varphi_{1}, \ldots, \varphi_{p}$ of $T$ we denote by $\left(\varphi_{1}, \ldots, \varphi_{p}\right)$ the automorphism fixing the uppermost layer of $T$, and acting by $\varphi_{i}$ on $S_{v_{i}}$, where $v_{i}$ is the $i$ th vertex in the uppermost layer. If $p=2$, then $G=\langle a, b, c, d\rangle$, where $a$ interchanges the points of level 1 , and $b=(a, c)$, $c=(a, d), d=(1, b)$. From this, we see that $G_{v}=\left\langle b, b^{a}, c, c^{a}, d, d^{a}\right\rangle$. Assume without loss of generality that $v$ is the right point on the first level. Then

$$
\rho\left(G_{v}\right)=\left\langle\rho(b), \rho\left(b^{a}\right), \rho(c), \rho\left(c^{a}\right), \rho(d), \rho\left(d^{a}\right)\right\rangle=\langle c, a, d, a, b, 1\rangle=G .
$$

For $p \geqslant 3$, we have $G=\langle a, b\rangle$, where $a$ is the cyclic permutation of the $p$ vertices of level 1 , and $b=\left(a, a^{-1}, 1, \ldots, 1, b\right)$. Now $G_{v}=\left\langle b, b^{a}, \ldots, b^{a^{p-1}}\right\rangle$. If we again assume that $v$ is the rightmost vertex, we obtain

$$
\rho\left(G_{v}\right)=\left\langle\rho(b), \rho\left(b^{a}\right), \ldots, \rho\left(b^{a^{p-1}}\right)\right\rangle=\left\langle b, a, a^{-1}, 1, \ldots, 1\right\rangle=G .
$$

In general, let $v$ be a vertex of level $\ell$ and $w$ the parent of $v$. The restriction of $G_{w}$ to the subtree with root $w$ is isomorphic to $G$ by the inductive hypothesis, and the restriction of $G_{v}$ equals the restriction of $\left(G_{w}\right)_{v}$. Since $v$ is of level 1 in the subtree with root $w$, the restriction of $G_{v}$ to the tree with root $v$ is isomorphic to $G$ by the case $\ell=1$ already proven.

On the other hand, we know that $G$ contains a subgroup $K$, such that $S t(\ell)$ contains $K^{\left(p^{\ell}\right)}$ geometrically, which is of index 16 or $p^{2}$, depending on whether $p=2$ or $p>2$. Hence, $\rho(S t(\ell)) \geqslant K$, and we conclude that $\left(\rho\left(G_{v}\right)\right.$ : $\rho(S t(\ell))) \leqslant p^{4}$.

In particular, we have the following. 
LeMma 22. Let $S$ be a complete tree and let $\varphi \in \operatorname{Inj}(S, T)$. Let $U<G$ be a subgroup of level at most $\ell$ and let $\mathcal{L}$ be the antichain of vertices of level $\ell$. We denote by $\rho$ the restriction of the action of $G$ from $\operatorname{Inj}(S, T)$ to $\operatorname{Inj}\left(S^{\mathcal{L}}, T^{\mathcal{L}}\right)$. If $S$ has exactly $p$ vertices of level $\ell$, then $\rho$ induces a surjection from the orbits of $U$ on $\varphi^{G}$ to the orbits of $U$ on $\rho(\varphi)^{G}$, and each pre-image under this injection contains at most $p^{4}$ elements.

Proof. As $\rho$ commutes with the action of $G$, it sends an orbit of $U$ on $\varphi^{G}$ to an orbit of $U$ on $\rho(\varphi)^{G}$; thus, the restriction to orbits is well defined. Since $\rho$ is surjective on $\operatorname{Inj}\left(S^{\mathcal{L}}, T^{\mathcal{L}}\right)$, it is also surjective on the orbits.

Let $v$ be the parent of the $p$ vertices of level $\ell$ in $\varphi(S)$, and let $\sigma$ be the restriction of the action of $G_{v}$ to the subtree $T_{v}$ of vertices below $v$. By Lemma 21, we have $\left(\sigma\left(G_{v}\right): \sigma(S T(\ell))\right) \leqslant p^{4}$; let $g_{1}, \ldots, g_{k}, k \leqslant p^{4}$ be elements of $G_{v}$, such that $\left\{\sigma\left(g_{1}\right), \ldots, \sigma\left(g_{k}\right)\right\}$ is a right transversal of $\sigma(S t(\ell))$ in $\sigma\left(G_{v}\right)$.

Suppose $\rho(\varphi)^{g}=\rho(\varphi)$; then $v^{g}=v$ as there are exactly $p$ vertices of level $\ell$ in $\varphi(S)$, and therefore, their parent is unique. Suppose that $g \in G$ satisfies $\rho(\varphi)^{g}=\rho(\varphi)^{u}$ for some $u \in U$. Then $g u^{-1} \in G_{v}$; hence, there exists some $i_{g}$ such that $\sigma\left(g u^{-1} g_{i_{g}}^{-1}\right) \in \sigma(S t(\ell))$. We claim that if $g, g^{\prime}$ are elements in $G$ with $\rho(\varphi)^{g}, \rho(\varphi)^{g^{\prime}} \in \rho(\varphi)^{U}$ and $i_{g}=i_{g^{\prime}}$, then $\varphi^{g} \in \varphi^{g^{\prime} U}$. Write $i=i_{g}=i_{g^{\prime}}$. By our argument above, there are $u, u^{\prime} \in U$, such that $\rho(\varphi)^{g u^{-1}}=\rho(\varphi)=\rho(\varphi)^{g u^{\prime-1}}$ and $\sigma\left(g u^{-1} g_{i}^{-1}\right), \sigma\left(g^{\prime} u^{\prime-1} g_{i}^{-1}\right) \in \sigma(S t(\ell))$. Hence, $\rho(\varphi)^{g u^{-1} g_{i}^{-1}}=\rho(\varphi)^{g^{\prime} u^{\prime-1}} g_{i}^{-1}$. Take $s \in S t(\ell)$ such that $\sigma\left(g u^{-1} g_{i}^{-1}\right)=\sigma\left(g^{\prime} u^{\prime-1} g_{i}^{-1} s\right)$. Then we also have that $\rho(\varphi)^{g u^{-1} g_{i}^{-1}}=\rho(\varphi)^{g^{\prime} u^{\prime-1} g_{i}^{-1} s}$. Since $S$ is contained in $T^{\mathcal{L}} \cup T_{v}$, we conclude $\varphi^{g u^{-1} g_{i}^{-1}}=\varphi^{g^{\prime} u^{\prime-1} g_{i}^{-1} s}$, so $\varphi^{g}=\varphi^{g^{\prime} u^{\prime-1} g_{i}^{-1} s g_{i} u}$. As $S t(\ell)$ is normal in $G$, we have that $s^{\prime}=g_{i}^{-1} s g_{i} \in S t(\ell)$, so $\varphi^{g}=\varphi^{g^{\prime} u^{\prime-1} s^{\prime} u}$. As $S t(\ell) \leqslant U$, we deduce that $\varphi^{g}=\varphi^{g^{\prime} u^{\prime \prime}}$ for some $u^{\prime \prime} \in U$.

We conclude that the number of orbits $\Omega$ of $U$ on $\varphi^{G}$, such that $\rho(\Omega)=\rho(\varphi)^{U}$ is at most equal to the number of possible choices of $i$, which is $k \leqslant p^{4}$. By symmetry, the same holds true for the other orbits of $U$ on $\rho(\varphi)^{G}$, and our claim follows.

LEMMA 23. Suppose that $S^{\prime} \subset S \subseteq T$ are complete trees, such that $S \backslash S^{\prime}$ consists of $p$ vertices, which are siblings of each other. Pick $\varphi \in \operatorname{Inj}(S, T)$, and let $\rho(\varphi) \in \operatorname{Inj}\left(S^{\prime}, T\right)$ be its restriction to $S^{\prime}$. Let $U$ be a subgroup of finite index of $G$. Write $N(U)$ for the number of orbits of $U$ on $\varphi^{G}$ and $N^{\prime}(U)$ for the number of orbits of $U$ on $\rho(\varphi)^{G}$. Then $N^{\prime}(U) \leqslant N(U) \leqslant p N^{\prime}(U)$.

Proof. Clearly, $\rho$ commutes with the action of $G$; hence, $\rho$ maps $U$-orbits to $U$ orbits. Since every map $S^{\prime} \rightarrow T$ can be extended to a map $S \rightarrow T, \rho$ is surjective on the set of orbits, and we obtain $N(U) \geqslant N^{\prime}(U)$. 
As $G$ is a $p$-group, the image of any subgroup of it in the symmetric group of $S \backslash S^{\prime}$ is either trivial or cyclic of order $p$. Therefore, for $g \in G$, the preimage of an orbit $\left(\rho\left(\varphi^{g}\right)\right)^{U}$ consists of 1 orbit or $p$ orbits. We conclude that $N(U) \leqslant p N^{\prime}(U)$, and the proof is complete.

LEMMA 24. Let $U<G$ be a subgroup of index $p^{n}$ and level at most $\ell$. Let $\mathcal{L}$ be the antichain consisting of all vertices of level $\ell$. Assume that $S$ is complete, that $S^{\mathcal{L}}$ contains $k$ vertices, and that $|S \cap \mathcal{L}|=p$. Pick $\varphi \in \operatorname{Inj}(S, T)$. Then $U$ acts with at most $n p^{\frac{k-p \ell-1}{p}+9}$ orbits on $\varphi^{G}$.

Proof. By Lemma 22, we have that the restriction $\rho: \operatorname{Inj}(S, T) \mapsto \operatorname{Inj}\left(S^{\mathcal{L}}, T^{\mathcal{L}}\right)$ induces a surjection from the set of orbits of $U$ on $\varphi^{G}$ to the set of orbits of $U$ on $\rho(\varphi)^{G}$, which is at most $p^{4}: 1$. It therefore suffices to prove that $U$ acts with at most $n p^{\frac{k-p \ell-1}{p}+5}$ orbits on $\rho(\varphi)^{G}$.

To avoid repeating arguments, we would like to use Theorem 7 again. Therefore, as our claim does not depend on the shape of $S$ below level $\ell$, we do not estimate the number of orbits on $\rho(\varphi)^{G}$, but assume without loss of generality that below level $\ell, S$ consists of an infinite path and the siblings of the vertices on this infinite path.

We now prove our claim by induction on $k$. On each of the first $\ell$ levels, $S$ has at least $p$ points. Together with the root we find that $k \geqslant \ell p+1$, and $k=\ell p+1$ if and only if $S$ is the completion of a single infinite path. In this case, $S$ has no automorphisms, that is, the actions of $G$ on $\varphi^{G}$ and on $S^{G}$ are equivalent. By Lemma 20(2), we may further restrict attention to a single path without completion. We can now invoke Theorem 7 to find that the number of orbits of $U$ on $\varphi^{G}$ is at most $p^{5} n$.

Now suppose that $k>\ell p+1$ and our claim holds for all trees $S^{\prime}$ such that $S^{\prime} L$ has less than $k$ vertices. Since $k>\ell p+1$, then $S$ is not a completion of a single infinite path. Therefore, there exists a vertex of level at most $\ell$ such that its parent is not on the infinite path. Let $x_{1}$ be such a vertex of maximal level. Then $x_{1}$ and its siblings $x_{2}, \ldots, x_{p}$ are leaves in $S$. Let $S^{\prime}$ be the tree that is obtained from $S$ by removing $x_{1}, \ldots, x_{p}$. Pick a map $\varphi \in \operatorname{Inj}(S, T)$, and let $\varphi^{\prime} \in \operatorname{Inj}\left(S^{\prime}, T\right)$ be its restriction.

By Lemma $23 N(U)$, the number of orbits of $U$ on $\varphi^{G}$ is at most $p$ times $N^{\prime}(U)$ the number of orbits of $U$ on $\varphi^{\prime G}$. By our inductive hypothesis,

$$
N^{\prime}(U) \leqslant n p^{\frac{\left|S_{\mathcal{L}}^{\prime}\right|-\ell p-1}{p}+5}=n p^{\frac{\left|S_{\mathcal{L}}\right|-p-\ell p-1}{p}+5}=n p^{\frac{k-\ell p-1}{p}+4},
$$

and our claim follows. 
Proof of Theorem 19. Let $f: \mathbb{N} \rightarrow \mathbb{N}$ be a function, and suppose that $\frac{f(n)}{n} \rightarrow \infty$, $\frac{f(n)}{p^{n}} \rightarrow 0$. We will construct a tree $S$ together with an increasing sequence of integers $\left(n_{i}\right)$, such that for $\varphi \in \operatorname{Inj}(S, T)$ the orbit growth of $G$ on $\varphi^{G}$ is strictly bounded by $f$, and for all $i$ there exist some $m_{i} \in\left[n_{i-1}, n_{i}\right]$ and a subgroup $U_{i}<G$ of index $p^{m_{i}}$, such that $U_{i}$ has at least $\frac{f\left(m_{i}\right)}{p}$ orbits on $\varphi^{G}$.

We will construct recursively a sequence of complete subtrees $S_{i}$ with $S_{i} \subset$ $S_{i+1}$ for all $i$ and $S_{i} \backslash S_{1}$ is finite and a sequence of maps $\varphi_{i} \in \operatorname{Inj}\left(S_{i}, T\right)$, such that $\left.\varphi_{i}\right|_{S_{i-1}}=\varphi_{i-1}$. Our construction will satisfy that there exists a strictly increasing sequence of integers $\left(n_{i}\right)$ with the following properties:

(i) For all $n \geqslant n_{1}$, the action of $G$ on $\varphi_{i}^{G}$ satisfies $o_{p^{n}}\left(G, \varphi_{i}^{G}\right) \leqslant f(n)$.

(ii) For all $k \geqslant 2$, there exist some $m_{k} \in\left[n_{k-1}, n_{k}\right]$ and a subgroup $U_{k}$ of index $p^{m_{k}}$, such that for all $i \geqslant k$ we have that $U_{k}$ acts on $\varphi_{i}^{G}$ with at least $\frac{f\left(m_{k}\right)}{p}$ orbits.

We let $S_{1}$ be the completion of some infinite path. As $S_{1}$ has no automorphisms, any map in $\operatorname{Inj}\left(S_{1}, T\right)$ can be identified with its image and thus, we can identify $\varphi_{1}^{G}$ with $S_{1}^{G}$. By Theorem 7 and the assumption that $\frac{f(n)}{n} \rightarrow \infty$, we can choose $n_{1}$ in such a way that for all $n \geqslant n_{1}, o_{p^{n}}\left(G, \varphi_{1}^{G}\right) \leqslant f(n)$.

Suppose we constructed $S_{j}$ and $n_{j}$ for all $j<i$. If we add vertices to $S_{i-1}$, then from Lemma 23 the orbit growth cannot decrease. Let $\ell$ be the maximum between the maximal level of a subgroup of index at most $p^{n_{i-1}}$ and the maximal level of a vertex in $S_{i-1} \backslash S_{1}$. If we only add vertices below level $\ell$ to $S_{i-1}$, then the orbit growth up to $n_{i-1}$ does not change. Write $I(m)=(G: S t(m))$. We would like to pick a number $\ell^{\prime}>\ell+2$, such that

$$
\text { for all } t \geqslant I\left(\ell^{\prime}-\ell\right) \text { we have } \frac{t}{p I(\ell)}>f(\log t) .
$$

To see that such an integer $\ell^{\prime}$ exists, assume that there exist arbitrarily large $t$, such that $\frac{t}{p I(\ell)} \leqslant f(\log t)$. Then for arbitrarily large $n=\log t$, we have $f(n) \geqslant$ $\frac{p^{n}}{p I(\ell)}$, contrary to the assumption $\frac{f(n)}{p^{n}} \rightarrow 0$. Hence, there exists some $t_{0}$, such that $\frac{t}{p I(\ell)}>f(\log t)$ holds for all $t>t_{0}$. As $I\left(\ell^{\prime}-\ell\right)$ tends to infinity with $\ell^{\prime}$, we can pick any $\ell^{\prime}$ such that $I\left(\ell^{\prime}-\ell\right)>t_{0}$.

Since $f$ grows faster than linearly, we can choose $n_{i}$ such that for all $n>n_{i}$,

$$
n p^{\frac{p^{\ell^{\prime}-\ell}-1}{p(p-1)}+\frac{p^{\ell+1}-1}{p(p-1)}+9}<f(n) .
$$

The reason for these choices will become clear below; for now, it is sufficient to picture these numbers as much larger than $\ell$ and $n_{i-1}$, respectively. 
We will construct $S_{i}$ by adding vertices to $S_{i-1}$ between levels $\ell+2$ and $\ell^{\prime}$. More specifically, let $v_{i}$ be the unique vertex of level $\ell+1$ on the infinite path of $S_{1}$. Then define $\mathcal{S}$ as the set of all complete trees $R$ containing $S_{i-1}$ and such that all vertices of $R \backslash S_{i-1}$ are descendants of $v_{i}$ and have level at most $\ell^{\prime}$. We will eventually choose $S_{i}$ within $\mathcal{S}$.

We first note that from the choice of $\ell$ it follows that for all $n \leqslant n_{i-1}$ and for all $R \in \mathcal{S}$, we have that $o_{p^{n}}\left(G, \varphi_{R}^{G}\right)=o_{p^{n}}\left(G, \varphi_{i-1}^{G}\right)$, where $\varphi_{R} \in \operatorname{Inj}(R, T)$ is an extension of $\varphi_{i-1}$. On the other hand, we claim that $o_{p^{n}}\left(G, \varphi_{R}^{G}\right)<f(n)$ for all $n>n_{i}$ and all $R \in \mathcal{S}$. To see this, note that for $R \in \mathcal{S}$, all vertices in $R \backslash S_{i-1}$ are descendants of $v_{i}$, and therefore, $\left|R \backslash S_{i-1}\right| \leqslant \frac{p^{\ell^{\prime}-\ell}-1}{p-1}$, and $\left|R \backslash S_{1}\right| \leqslant$ $\frac{p^{\ell^{\prime}-\ell}-1}{p-1}+\frac{p^{\ell+1}-1}{p-1}$.

Let $U$ be a subgroup of index $p^{n}$ and pick $\lambda>\ell^{\prime}$ such that $\lambda$ is greater than the level of $U$. Let $\mathcal{L}$ be the antichain consisting of all vertices on level $\lambda$ and let $k=\left|R^{\mathcal{L}}\right|$. Since $\lambda>\ell^{\prime}$, then $|R \cap \mathcal{L}|=p$. We can now apply Lemma 24 to find that the number of orbits of $U$ on $\varphi_{R}^{G}$ is at most $n p^{\frac{k-p \lambda-1}{p}+9}$. Now,

$$
\begin{aligned}
k=\left|R^{\mathcal{L}}\right|=\left|\left(S_{1}\right)^{\mathcal{L}}\right|+\left|\left(R \backslash S_{1}\right)^{\mathcal{L}}\right| & \leqslant\left|\left(S_{1}\right)^{\mathcal{L}}\right|+\left|R \backslash S_{1}\right| \\
& \leqslant p \lambda+1+\frac{p^{\ell^{\prime}-\ell}-1}{p-1}+\frac{p^{\ell+1}-1}{p-1},
\end{aligned}
$$

so

$$
k-p \lambda-1 \leqslant \frac{p^{\ell^{\prime}-\ell}-1}{p-1}+\frac{p^{\ell+1}-1}{p-1} .
$$

Therefore, the number of orbits of $U$ on $\varphi_{R}^{G}$ is at most

$$
n p^{\frac{k-p \lambda-1}{p}+9} \leqslant n p^{\frac{p^{\ell}-\ell-1}{p(p-1)}+\frac{p^{\ell+1}-1}{p(p-1)}+9} .
$$

By the choice of $n_{i}$ in (2), we deduce that $o_{p^{n}}\left(G, R^{G}\right)<f(n)$ for all $n>n_{i}$ as claimed. We conclude that condition (i) holds for all $R \in \mathcal{S}$ and all $n$ satisfying $n \leqslant n_{i-1}$ or $n>n_{i}$.

Call a tree $R \in \mathcal{S}$ small, if the action of $G$ on $\varphi_{R}^{G}$ has orbit growth bounded by $f(n)$ for all $n_{i-1}<n \leqslant n_{i}$, and large otherwise. We know that $S_{i-1}$ itself is small. Next we claim that a large tree exists. To see this, take the tree $S_{\max }$, consisting of $S_{i-1}$ together with all vertices of level $\leqslant \ell^{\prime}$, which lie below $v_{i}$. Let $\Delta$ be all the descendants of $v_{i}$ of level $\ell^{\prime}$. Let $\mathcal{A}$ be the antichain in $T$ consisting of all vertices of level $\ell$ except the parent of $v_{i}$, the $p-1$ siblings of $v_{i}$, and $\Delta$. Since all vertices in $\mathcal{A}$ are of level at most $\ell$, we have that $G_{\mathcal{A}} \leqslant S t(\ell)$. Also, $G_{\mathcal{A}}$ acts trivially on $\Delta$ as $\Delta \subseteq \mathcal{A}$.

Pick $\varphi_{\max } \in \operatorname{Inj}\left(S_{\max }, T\right)$, and put $Y=\varphi_{\max }^{S t(\ell)}$. Then $Y$ is $G_{\mathcal{A}}$-invariant as $G_{\mathcal{A}} \leqslant$

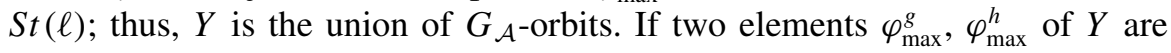


in the same $G_{\mathcal{A}}$-orbit, then $\varphi_{\max }^{g}(x)=\varphi_{\max }^{h}(x)$ holds for all $x \in \Delta$ because $G_{\mathcal{A}}$ acts trivially on $\Delta$. Therefore, the number of different orbits of $G_{\mathcal{A}}$ on $Y$ is at least equal to the number of permutations of $\Delta$, which occur as restrictions of elements of $S t(\ell) \cap G_{v_{i}}$. The kernel of the map $\left(S t(\ell) \cap G_{v_{i}}\right) \rightarrow \operatorname{Sym}(\Delta)$ is $G_{\mathcal{A}}$; therefore, the number of orbits of $G_{\mathcal{A}}$ is at least

$$
\left(\left(S t(\ell) \cap G_{v_{i}}\right): G_{\mathcal{A}}\right)=\frac{\left(G: G_{\mathcal{A}}\right)}{(G: S t(\ell))\left(\operatorname{St}(\ell):\left(S t(\ell) \cap G_{v_{i}}\right)\right)}=\frac{\left(G: G_{\mathcal{A}}\right)}{p I(\ell)} .
$$

Let $T_{v_{i}}$ be the subtree of $T$ with root $v_{i}$, and let $\rho$ be the restriction of $G_{v_{i}}$ to $T_{v_{i}}$. By Lemma 21 , we have $\rho\left(G_{v_{i}}\right) \cong G$ as permutation groups and by definition, $\rho\left(G_{\mathcal{A}}\right) \cong S t\left(\ell^{\prime}-\ell\right)$. Then

$$
\left(G: G_{\mathcal{A}}\right) \geqslant\left(G_{v_{i}}: G_{\mathcal{A}}\right) \geqslant\left(\rho\left(G_{v_{i}}\right): \rho\left(G_{\mathcal{A}}\right)\right)=\left(G: S t\left(\ell^{\prime}-\ell\right)\right)=I\left(\ell^{\prime}-\ell\right) .
$$

In particular, we can apply the definition of $\ell^{\prime}$ in (1) with $t=\left(G: G_{\mathcal{A}}\right)$, as the condition $t \geqslant I\left(\ell^{\prime}-\ell\right)$ is satisfied. Doing so, we obtain

$$
o_{\left(G: G_{\mathcal{A}}\right)}\left(G, \varphi_{\max }^{G}\right) \geqslant\left(\left(S t(\ell) \cap G_{v_{i}}\right): G_{\mathcal{A}}\right)=\frac{\left(G: G_{\mathcal{A}}\right)}{p I(\ell)}>f\left(\log \left(G: G_{\mathcal{A}}\right)\right),
$$

that is, $S_{\max }$ is large.

Define a graph on $\mathcal{S}$ by drawing an edge between two subtrees if one of them can be obtained from the other by adding or deleting one vertex and its siblings. The graph is connected as every tree in $\mathcal{S}$ is connected to $S_{i-1}$. Since there are small and large trees, there exists a small tree $S_{i}$, which is connected to a large tree $S_{i}^{+}$. As $S_{i}$ is small, it satisfies condition (i).

Since $S_{i}^{+}$is large, there exist some $n_{i-1} \leqslant m_{i}<n_{i}$ and a subgroup $U_{i}$ of index $p^{m_{i}}$, which acts with more than $f\left(m_{i}\right)$ orbits on $\left(\varphi_{i}^{+}\right)^{G}$ for some $\varphi_{i}^{+} \in \operatorname{Inj}\left(S_{i}^{+}, T\right)$. Since $S_{i}$ and $S_{i}^{+}$are complete, we can apply Lemma 23 and find that the number of orbits of $U_{i}$ on $\left(\varphi_{i}^{+}\right)^{G}$ is at most $p$ times the number of orbits of $U_{i}$ on $\varphi_{i}^{G}$, where $\varphi_{i}$ is the restriction of $\varphi_{i}^{+}$to $S_{i}$. In particular,

$$
o_{p^{m_{i}}}\left(G, \varphi_{i}^{G}\right) \geqslant \frac{1}{p} o_{p^{m_{i}}}\left(G, \varphi_{i}^{+G}\right)>\frac{1}{p} f\left(m_{i}\right) .
$$

We conclude that $S_{i}$ satisfies condition (ii) as well.

Now define the tree $S=\bigcup S_{i}, \varphi=\bigcup \varphi_{i}$. It follows from Lemma 23 that the number of orbits of a finite index subgroup $U$ on $\varphi^{G}$ is at least equal to the number of orbits of $U$ on $\varphi_{i}^{G}$ for any $i$. On the other hand, by the construction of the trees $S_{i}$, we know that there are arbitrarily large values $\ell$, such that the intersection of $S$ with the $\ell$ th level consists of $p$ points only. For each of these levels, we can apply Lemma 22 to find that the number of orbits of $U$ 
on $\varphi^{G}$ is at most $p^{4}$ times the number of orbits of $U$ on $\varphi_{i}^{G}$, provided that $i$ is sufficiently large. We conclude that the action of $G$ on $\varphi^{G}$ satisfies the conditions of Theorem 19, and the theorem is proven.

\section{Acknowledgements}

We thank Alejandra Garrido for reading carefully a previous version of this paper and making an abundance of useful comments. We thank Alejandra Garrido and Benjamin Klopsch for organizing and inviting us to the conference 'Trees, dynamics, and locally compact groups', where a significant part of this research took place. We thank the Alexander von Humboldt Foundation and the Deutsche Forschungsgemeinschaft for supporting this conference. We thank the anonymous referee for his/her careful reading and his/her valuable comments.

\section{Conflict of Interest: None.}

\section{References}

[1] T. Alexoudas, B. Klopsch and A. Thillaisundaram, 'Maximal subgroups of multi-edge spinal groups', Groups Geom. Dyn. 10 (2016), 619-648.

[2] M. Ershov and A. Jaikin-Zapirain, 'Groups of positive weighted deficiency and their applications', J. Reine Angew. Math. 677 (2013), 71-134.

[3] G. Fernandez-Alcober and A. Zugadi-Reizabal, 'GGS-groups: order of congruence quotients and Hausdorff dimension', Trans. Amer. Math. Soc. 366 (2014), 1993-2017.

[4] A. Garrido, 'Abstract commensurability and the Gupta-Sidki group', Groups Geom. Dyn. 10 (2016), 523-543.

[5] R. I. Grigorchuk, 'Just infinite branch groups', in New Horizons in pro-p Groups (eds. M. du Sautoy, D. Segal and A. Shalev) Birkhäuser Progress in Mathematics, 184 (Birkhäuser Verlag, Basel, 2000), 121-179.

[6] R. I. Grigorchuk and J. S. Wilson, 'A structural property concerning abstract commensurability of subgroups', J. Lond. Math. Soc. (2) 68 (2003), 671-682.

[7] B. Klopsch, 1999 'Substitution Groups, Subgroup Growth and Other Topics', D.Phil. Thesis, University of Oxford.

[8] A. Lubotzky and D. Segal, Subgroup Growth, Birkhäuser Progress in Mathematics, 212 (Birkhäuser Verlag, Basel, 2003).

[9] E. L. Pervova, 'Maximal subgroups of some non locally finite p-groups', Internat. J. Algebra Comput. 15 (2005), 1129-1150.

[10] L. Pyber, 'Groups of intermediate subgroup growth and a problem of Grothendieck', Duke Math. J. 121 (2004), 169-188.

[11] D. Segal, 'The finite images of finitely generated groups', Proc. Lond. Math. Soc. (3) 82 (2001), 597-613.

[12] D. Segal and A. Shalev, 'Groups with fractionally exponential subgroup growth', J. Pure Appl. Algebra 88 (1993), 205-223. 
[13] S. Sidki, 'On a 2-generated infinite 3-group: subgroups and automorphisms', J. Algebra 110 (1987), 24-55.

[14] A. Shalev, 'Growth functions $p$-adic analytic groups and groups of finite coclass', J. Lond. Math. Soc. (2) 46 (1992), 111-122.

[15] A. Zugadi-Reizabal, 2011 'Hausdorff dimension in groups acting on the $p$-adic tree', $\mathrm{PhD}$ Thesis, University of the Basque country Bilbao. 